\title{
Characteristics and spread to the native population of HIV-1 non-B subtypes in two European countries with high migration rate
}

Kenny Dauwe ${ }^{1}$, Virginie Mortier ${ }^{1}$, Marlies Schauvliege ${ }^{1}$, Annelies Van Den Heuvel ${ }^{2}$, Katrien Fransen², Jean-Yves Servais ${ }^{3}$, Danielle Perez Bercoff ${ }^{3}$, Carole Seguin-Devaux ${ }^{3}$ and Chris Verhofstede ${ }^{1 *}$

\begin{abstract}
Background: Non-B subtypes account for at least $50 \%$ of HIV-1 infections diagnosed in Belgium and Luxembourg. They are considered to be acquired through heterosexual contacts and infect primarily individuals of foreign origin. Information on the extent to which non-B subtypes spread to the local population is incomplete.

Methods: Pol and env gene sequences were collected from 410 non-subtype B infections. Profound subtyping was performed using 5 subtyping tools and sequences of both pol and env. Demographic information, disease markers (viral load, CD4 count) and viral characteristics (co-receptor tropism) were compared between subtypes. Maximum likelihood phylogenetic trees were constructed and examined for clustering.

Results: The majority of non-B infections were diagnosed in patients originating from Africa (55.8\%), individuals born in Western Europe represented $30.5 \%$. Heterosexual transmission was the most frequently reported transmission route (79.9\%), MSM transmission accounted for $12.2 \%$ and was significantly more frequently reported for Western Europeans ( $25.7 \%$ versus $4.3 \%$ for individuals originating from other regions; $p<0.001$ ). Subtypes A and $C$ and the circulating recombinant forms CRF01_AE and CRF02_AG were the most represented and were included in the comparative analysis. Native Western Europeans were underrepresented for subtype A (14.5\%) and overrepresented for CRF01_AE (38.6 \%). The frequency of MSM transmission was the highest for CRF01_AE (18.2\%) and the lowest for subtype A (0 \%). No differences in age, gender, viral load or CD4 count were observed. Prevalence of CXCR4-use differed between subtypes but largely depended on the tropism prediction algorithm applied. Indications for novel intersubtype recombinants were found in 20 patients (6.3\%). Phylogenetic analysis revealed only few and small clusters of local transmission but could document one cluster of CRF02_AG transmission among Belgian MSM.
\end{abstract}

Conclusions: The extent to which non-B subtypes spread in the native Belgian-Luxembourg population is higher than expected, with $30.5 \%$ of the non-B infections diagnosed in native Western Europeans. These infections resulted from hetero- as well as homosexual transmission. Introduction of non-B variants in the local high at risk population of MSM may lead to new sub-epidemics and/or increased genetic variability and is an evolution that needs to be closely monitored.

Keywords: HIV-1 non-B infections, HIV-1 subtyping, Subtyping tools, Non-B HIV-1 and co-receptor use, Non-B spread in Western Europe

\footnotetext{
* Correspondence: chris.verhofstede@ugent.be

'Aids Reference Laboratory, Department of Clinical Chemistry, Microbiology and Immunology, Ghent University, De Pintelaan 185-Blok A, B-9000, Ghent, Belgium

Full list of author information is available at the end of the article
}

\section{Biomed Central}

(c) 2015 Dauwe et al. Open Access This article is distributed under the terms of the Creative Commons Attribution 4.0 International License (http://creativecommons.org/licenses/by/4.0/), which permits unrestricted use, distribution, and reproduction in any medium, provided you give appropriate credit to the original author(s) and the source, provide a link to the Creative Commons license, and indicate if changes were made. The Creative Commons Public Domain Dedication waiver (http://creativecommons.org/publicdomain/zero/1.0/) applies to the data made available in this article, unless otherwise stated. 


\section{Background}

High virus replication rates and error prone reverse transcription resulted in the genetic diversification of the main HIV-1 group $M$ into 9 subtypes: A, B, C, D, F, G, $\mathrm{H}, \mathrm{J}$ and $\mathrm{K}$ [1]. The possibility of recombination further fuels genetic variability. Amongst the constantly increasing number of circulating recombinant forms (CRF), CRF01_AE and CRF02_AG have been the most successful (http://www.hiv.lanl.gov/content/sequence/HIV/CRFs/ CRFs.html; last accessed on December $4^{\text {th }} 2014$ ) [2]. Between 2004 and 2007, the subtypes $C$ and A, together with the recombinants CRF01_AE and CRF02_AG were responsible for $73 \%$ of all infections worldwide [2]. Since the beginning of the HIV epidemic, non-B infections accounted for up to $50 \%$ of all new diagnoses in Belgium and Luxembourg $[3,4]$. Today, the proportion of non-B infections in Luxembourg may reach $62 \%$ [5]. Non-B infections are mainly diagnosed in individuals of foreign origin and in general acquired through heterosexual contact. This contrasts with the epidemic in the local population that is almost exclusively driven by men having sex with men (MSM) infected with subtype B virus. Recent analysis of transmission dynamics in Luxembourg however provided evidence for an increasing number of established non-B transmissions in the country [5]. Subtype specific differences in disease progression, transmission efficiency or susceptibility to antiretroviral drugs have been reported but there still is a lack of consistency in these data $[6,7]$.

Adequate subtyping is essential when studying the distribution and characteristics of the different non-B subtypes. While the initial classification of HIV-1 into subtypes was based on full length sequences most epidemiological and clinical studies today, for practical reasons, use partial sequences for subtyping. Polymerase ( $p o l)$ gene sequences are preferably used for that purpose because they are widely available through routine drug resistance screening and are considered to contain sufficient phylogenetic signal for subtype assignment [8]. Subtype classification based on one genomic region however, may miss intersubtype recombination [9]. Single region subtyping therefore can introduce bias, especially when studying viral characteristics not defined by the pol gene such as co-receptor use. The preference of HIV-1 for one of the two co-receptors, CCR5 and CXCR4, is determined by a small variable region (V3) of the envelope (env) gene. The prevalence of CXCR4-use seems to differ between subtypes with low CXCR4-use reported for subtype $C$ and high CXCR4-use for subtype D and CRF01_AE [10-18]. In subtype B, the ability to use CXCR4 has been associated with faster disease progression $[19,20]$. For the other subtypes, the potential association between co-receptor use and disease progression is less well documented.

The study presented here is a retrospective analysis comparing the characteristics of non-B HIV-1 infections in two countries with low HIV prevalence but with a high diversity of subtypes [3, 4, 21]. Extended subtyping of 410 non-B infections was performed and patient demographics, HIV transmission route, co-receptor use, viral load and CD4 count were compared between subtypes. The extent to which the non-B subtypes spread in the native population was defined.

\section{Methods}

\section{Patients and sequences}

HIV-1 pol sequences (857 nucleotides (nt) long concatenated sequence fragments comprising codon 4 to 99 of the Protease gene and codon 30 to 226 of the Reverse transcriptase gene), were collected from 410 non-B subtype HIV-1 infected individuals diagnosed at two sites in Belgium (Ghent, 277 patients; Antwerp, 82 patients) and one site in Luxembourg (51 patients) between 2000 and 2012. The pol sequences were obtained as part of routine clinical care for baseline or pretreatment resistance analysis. Initial identification of non-B infection was based on the fast subtyping tool implemented in the Smartgene IDNS database system (Smartgene IDNS, Zug, Switzerland). Selected patients had to be treatment naïve and have sufficient left over plasma available for env sequencing. Env sequences were generated following the procedures for RNA extraction, amplification and Sanger sequencing as described before [22]. This procedure generates an amplicon of about $1106 \mathrm{nt}$ long comprising V1 to V4. The short V3 sequence, with a length of $105 \mathrm{nt}$, could be generated for all 410 samples but sequencing of a longer env fragment (of at least $300 \mathrm{nt}$ ) was successful for only 337 samples due to high sequence variability and the abundant presence of indels in this region. Short V3 sequences were used for co-receptor tropism prediction only, the longer sequences were also used for subtype assignment. Demographic, epidemiological and clinical data were collected from the patients' records, including gender, age, origin, most probable route of transmission, CD4 count and viral load.

\section{Ethics statement}

The study was reviewed and approved by the Ethics Committee of Ghent University Hospital (as central committee; study number 2011/162), the Institute of Tropical Medicine Antwerp and the Comité National d'Ethique pour la Recherche in Luxembourg.

\section{Subtyping}

Subtyping was performed using a combination of 5 subtyping tools: Rega v3, http://regatools.med.kuleuven.be/ typing/v3/hiv/typingtool (Rega Institute for Medical Research, Leuven, Belgium) [23]; Comet, http://comet.retrovirology.lu/ (Laboratory of Retrovirology, Luxembourg 
Institute of Health, Luxembourg) [21]; SCUEAL, http:// www.datamonkey.org/dataupload_scueal.php (University of California San Diego, La Jolla, California, US) [24]; jpHMM, http://jphmm.gobics.de/submission_hiv (Institute of Microbiology and Genetics, University of Göttingen, Germany) [25] and NCBI, http://www.ncbi.nlm.nih.gov/projects/genotyping/formpage.cgi (National Center for Biotechnology Information, Bethesda, MD) [26]. To classify pol sequences, Comet and Rega were used initially and the subtype was attributed in case of concordant results. Sequences with discordant results or for which the subtype remained unassigned by at least one of the two tools were additionally submitted to SCUEAL and jpHMM. The final subtype was attributed in case of concordance for at least 3 tools. All other sequences were considered as unassigned. The same strategy was used for env subtyping but, given that the SCUEAL tool can only handle pol sequences it was replaced by NCBI.

\section{Genotypic tropism prediction}

V3 nucleotide sequences were submitted to geno2pheno (co-receptor) (G2P, http://coreceptor.geno2pheno.org, Max Planck Institute for Informatics, Saarbrücken, Germany) for co-receptor tropism prediction [27] and interpreted using the FPR cut off values of 10 and $5.75 \%$. V3 sequences were also submitted to Web PSSM (http://indra. mullins.microbiol.washington.edu/webpssm/, Department of Microbiology, University of Washington, Seattle, Washington, US) $[28,29]$ and run with the X4/R5 subtype $B$ matrix and for subtype $C$ also with the subtype C SI/NSI algorithm. For the subtypes A and C and CRF01_AE and CRF02_AG additional tropism prediction was performed with a recently developed subtype specific algorithm, Phenoseq (http://tools.burnet.edu.au/phenoseq/, Burnet Institute, Melbourne, Australia) [30].

\section{Phylogenetic analysis}

Pol sequences and env sequences $>300 \mathrm{bp}$, were aligned in the BioEdit Sequence Alignment Editor Version 7.0.9. For subtype verification, Los Alamos 2010 subtype and CRF reference sequences with exclusion of the subtype B and group P references (http://www.hiv.lanl.gov/cgi-bin/ NEWALIGN/align.cgi; last accessed on December $5^{\text {th }}$ 2014) were added to the alignment. For examination of origin-specific and transmission-route specific clustering the subtype references were excluded. The HXB2 sequence was used as outgroup in all trees. Maximum likelihood trees were generated using the 'best fit' evolutionary model selected by jModeltest version 2.1.7 [31] and the PhyML software package with approximate likelihood ratio test (aLRT) for branch support [32]. Tree visualization and editing was done with Itol v2.2.2 [33]. Clusters of presumed recent transmission were identified with the automated cluster selection tool developed recently by
Ragonnet-Cronin et al. [34] using as thresholds aLRT $>0.9$ and mean genetic distance $<0.045$.

\section{Statistical analyses}

Statistical analyses were performed using SPSS 22.0 software (IBM Corp. Released 2013. IBM SPSS Statistics for Windows, Version 22.0. Armonk, NY: IBM Corp). Bivariate analyses used the chi-square test or Fisher exact test (if more than $20 \%$ of the cells had expected counts less than 5) for categorical variables and the MannWhitney $\mathrm{U}$ nonparametric test for continuous variables. The level of significance was set at $p<0.05$. $P$-values were calculated to assess whether the analyzed parameters showed subtype specific differences. Characteristics of individual subtypes were compared to the pool of all other subtypes.

\section{Results}

Patients

A total of 410 individuals infected with non-B HIV-1 were selected, $47.3 \%$ were male and the mean age was 33.5 years. Of the 382 individuals with known origin, 213 (55.8 \%) were from Africa, 95 (24.9\%) from Belgium or Luxembourg, 22 (5.6 \%) from another Western European country, 14 (3.7 \%) from Eastern Europe, 32 (8.4 \%) from Asia, 5 from South America (1.3\%) and 1 from the Middle East $(0.3 \%)$. Of the 303 infections with known infection route, heterosexual transmission was the most frequently reported $(242 ; 79.9 \%)$ followed by MSM transmission (37; $12.2 \%$ ), intravenous drug use (IVDU) (10; $3.3 \%)$, blood transfusion $(7 ; 2.3 \%)$ and congenital infection (1;0.3\%). Multiple risk factors were reported by 6 individuals $(2.0 \%)$. Distribution of transmission route for the 184 individuals born outside of Western Europe was heterosexual contact $(161 ; 87.5 \%)$, MSM $(8 ; 4.3 \%)$, blood transfusion (5; $2.7 \%)$, IVDU (5; $2.7 \%)$, congenital transmission $(1 ; 0.5 \%)$ and combined risks $(4 ; 2.2 \%)$. Of the 109 individuals born in Western Europe with known infection route, transmission resulted from heterosexual contact in 73 (67.0\%), MSM in 28 (25.7\%), IVDU in 5 (4.6\%), blood transfusion in $2(1.8)$ and combined risks in 1 (0.9 \%). The distribution of MSM and heterosexual transmission differed significantly between the individuals born in Western Europe and those born elsewhere $(p<0.001)$.

\section{Subtyping}

Rega and Comet reported a concordant subtype for 351 $(85.6 \%)$ of the $410 \mathrm{pol}$ sequences. Ten of the 59 sequences with discordant or unassigned subtype could be attributed to a subtype after consulting jpHMM and SCUEAL, 49 (11.3 \%) remained unassigned. Subtype distribution was CRF02_AG (100; $27.7 \%)$, A (74; $20.5 \%)$, CRF01_AE (66; $18.3 \%)$, C (63; $17.5 \%)$, G (29; $8.0 \%)$, 
F (11; $3.0 \%), D(8 ; 2.2 \%)$, CRF06_cpx (4; $1.1 \%)$, CRF12_BF (2; $0.6 \%), \mathrm{H}$ (1;0.3\%), CRF03_AB (1; $0.3 \%)$, CRF11_cpx (1; $0.3 \%)$ and CRF37_cpx (1; $0.3 \%)$. Subtyping of the 318 env sequences with Rega and Comet resulted in concordant results for 230 (72.3\%). After consulting jpHMM and NCBI 76 additional sequences were subtyped, 12 (3.8 \%) remained unassigned. Because env does not allow to reliably discriminate between subtype A and CRF02_AG, both were classified as 'A-like'. Final subtype distribution for the env sequences was A-like (148; $48.4 \%)$, C (56; $18.3 \%)$, CRF01_AE (54; $17.6 \%)$, G $(21 ; 6.9 \%), \mathrm{B}(12 ; 3.9 \%), \mathrm{F}(8 ; 2.6 \%), \mathrm{D}(6 ; 2.0 \%)$ and $\mathrm{H}(1 ; 0.3 \%)$.

\section{Concordance between pol and env subtyping}

For comparative analysis of the pol and env subtyping, pol sequences classified as CRF03, CRF06, CRF11, CRF12, CRF13 and CRF37 were equalized to the subtype that constituted the env region; respectively B, G, A, F, A and A. Under these conditions, overall concordance between the pol and the env subtype was $81.1 \%$ (259/318) (Table 1). Lack of concordance resulted from discordant subtype attribution $(n=24)$ or failed subtyping for pol $(n=23)$, env $(n=4)$ or both $(n=8)$. When the 35 subtyping failures were excluded, overall concordance rose to $91.5 \%$. Of the 23 samples with unassigned pol but assigned env subtype, the env classification was A-like for 16 (69.5\%), G for 5 (21.7\%) and B for 2 (8.7\%). Twenty four patients (7.5\% of those with both pol and env subtype available) showed evidence of intersubtype recombination. The novel combinations were CRF02_AG/G $(n=4), \mathrm{G} / \mathrm{B}(n=4), \mathrm{A} / \mathrm{B}$ $(n=3), \mathrm{D} / \mathrm{A}(n=3), \mathrm{G} / \mathrm{A}(n=2), \mathrm{CRF} 02 \_\mathrm{AG} / \mathrm{B}(n=2)$, CRF01_AE/A $(n=1)$, CRF01_AE/C $(n=1)$, CRF02_AG/D $(n=1), \mathrm{A} / \mathrm{C}(n=1), \mathrm{A} / \mathrm{G}(n=1)$ and F/A $(n=1)$. Nine of these potential novel recombinants were classified as subtype B in env. Four were identified as CRF14_BG

Table 1 Comparison of the subtypes assigned for the pol and env sequences

\begin{tabular}{|c|c|c|c|c|c|c|c|c|c|c|}
\hline \multirow[b]{2}{*}{ Env subtype } & \multicolumn{10}{|c|}{ Pol subtype } \\
\hline & $\bar{A}$ & $B$ & $C$ & $\mathrm{D}$ & $F$ & G & $\mathrm{H}$ & 01_AE & 02_AG & $\cup A$ \\
\hline A-like & 59 & & & 3 & 1 & 3 & & 1 & 71 & 16 \\
\hline B & 3 & 1 & & & & 4 & & & 2 & 2 \\
\hline$C$ & 1 & & 62 & & & & & 1 & & \\
\hline$D$ & & & & 5 & & & & & 1 & \\
\hline$F$ & & & & & 6 & & & & & \\
\hline G & 1 & & & & & 12 & & & 5 & 5 \\
\hline $\mathrm{H}$ & & & & & & & 1 & & & \\
\hline CRF01AE & & & & & & & & 57 & & \\
\hline UA & & & & & & 2 & & & 2 & 8 \\
\hline
\end{tabular}

after phylogenetic analysis, 5 were novel non-B/B recombinants. When excluding CRF14_BG, 20 (6.3 \%) novel recombinants remained with 6 isolated from native Western Europeans.

\section{Phylogenetic and cluster analysis}

Manual examination of the pol phylogenetic tree (Fig. 1) showed marked subtype specific clustering. A large number of the pol sequences for which the subtyping tools failed were localized in the subtype A cluster or in the CRF02_AG cluster (respectively 16 and 9 of the 49). Sequences of individuals with Belgian or Luxembourg origin were scattered over the tree. They were however in the majority in the small subtype F cluster. An important representation of Western Europeans, mainly originating from Portugal was also observed for subtype G. In the large and very branched subtype A cluster, a marked clustering $(\mathrm{aLRT}=1.0$ ) of sequences from Eastern Europeans, many of whom reporting IVDU transmission, was identified (Fig. 1, cluster 1).

Using stringent cluster selection criteria (aLRT $>0.90$, mean genetic distance $<0.045$ and cluster composed of at least 3 individuals) the pol tree was further examined for indications of recent transmission events. Seven transmission clusters were identified; 5 clusters of 3 individuals, 1 of 4 individuals and 1 of 6 individuals. Five of these 7 clusters were also identified in the env tree (Fig. 2) although for some the env cluster size was smaller because not all individuals had an env sequence of $>300$ bp available. Six of the 7 transmission clusters resulted from heterosexual transmission. They were clusters of CRF01_AE (cluster 2 and 3 in Figs. 1 and 2), subtype A (cluster 4), CRF06_cpx (cluster 5), novel A/B recombinant (cluster 6) and unassigned sequences (cluster 7). The largest cluster (cluster 8) was a CRF02_AG cluster of 6 individuals of whom 5 reported MSM as transmission risk and 5 were of Western European origin.

\section{Subtype related differences in patient demographics, viral load, CD4 count, co-receptor tropism and transmission route}

To ensure sufficient sample numbers for statistical analysis, only the subtypes A and C and the CRF01_AE and CRF02_AG were considered as separate groups, other subtypes and CRF were pooled and sequences with unassigned subtype were excluded. The characteristics of the patients grouped after pol subtyping are shown in Table 2. No differences were observed in age or gender distribution, viral load or CD4 counts. Significant differences in the origin of patients were recorded: patients originating from Central and Western Africa and from Eastern Europe were more frequently infected with subtype A, individuals from Western Africa with CRF02_AG, individuals from Asia with CRF01_AE and individuals 


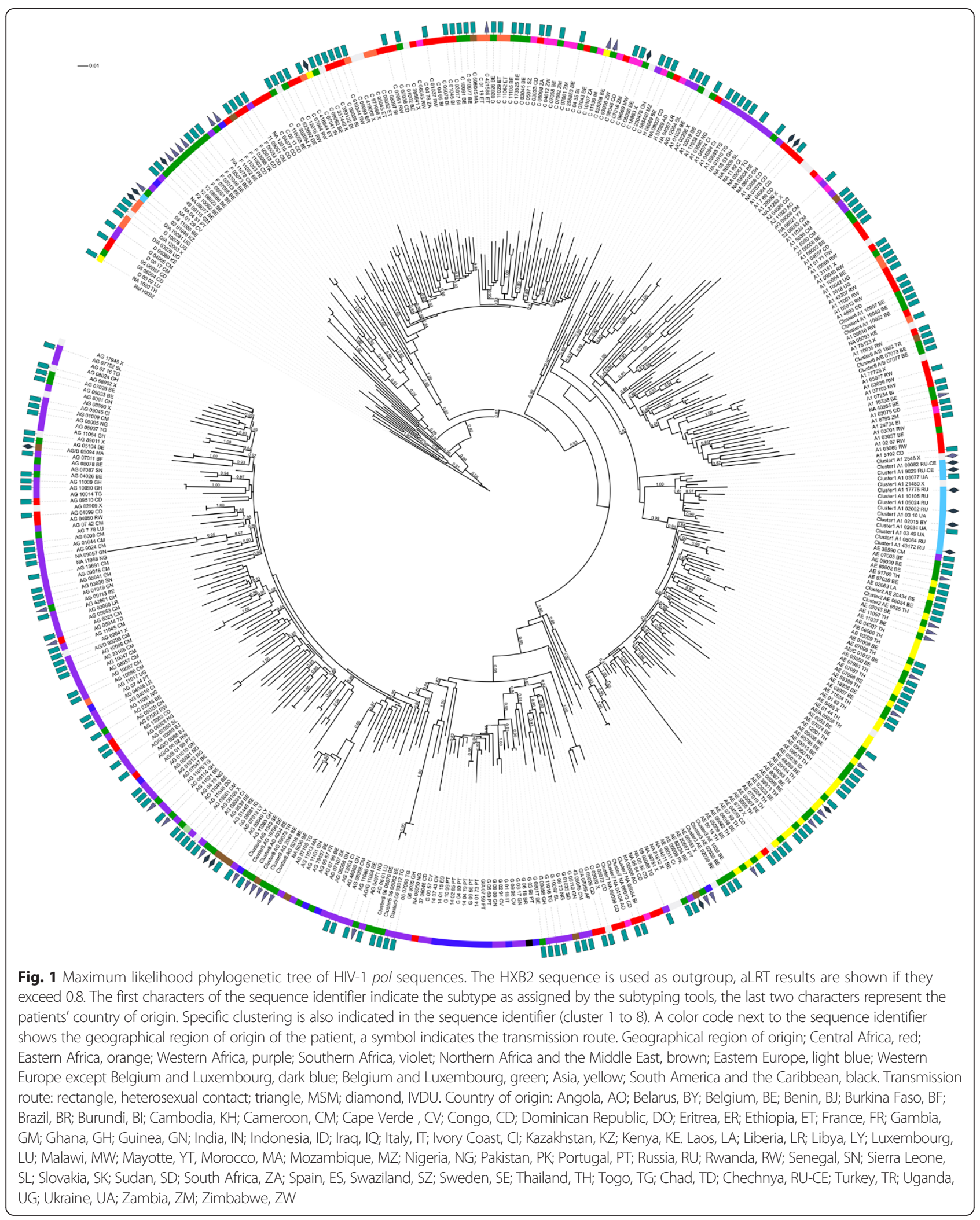




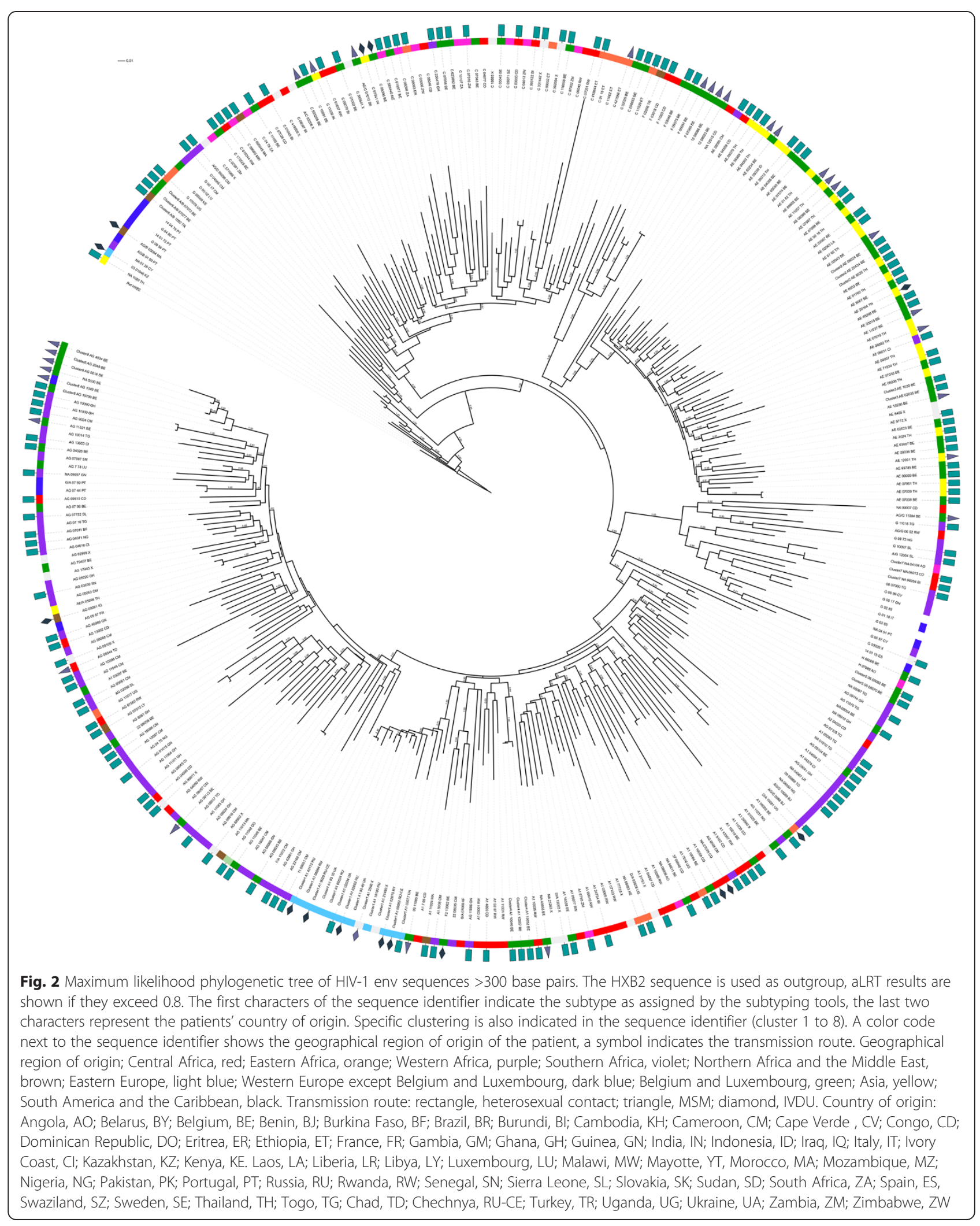


Table 2 Patient demographics, viral load, CD4 count, co-receptor tropism and transmission route for the most prevalent subtypes and CRF

\begin{tabular}{|c|c|c|c|c|c|c|c|c|c|c|c|}
\hline \multirow[b]{2}{*}{ Total, $N=366$} & \multicolumn{2}{|l|}{$\mathrm{A}(N=74)$} & \multicolumn{2}{|l|}{$C(N=63)$} & \multicolumn{2}{|c|}{ CRF_01AE $(N=66)$} & \multicolumn{2}{|c|}{ CRF02_AG $(N=100)$} & \multicolumn{2}{|c|}{ Other subtypes and CRF $(N=63)$} & \multirow[t]{2}{*}{$p$} \\
\hline & Count (\%) & $p$ & Count (\%) & $p$ & Count (\%) & $p$ & Count (\%) & $p$ & Count (\%) & $p$ & \\
\hline \multicolumn{12}{|l|}{ Gender, $N=301$} \\
\hline Male, N (\%) & $32(50)$ & NS & $22(38.6)$ & NS & $35(56.5)$ & 0.089 & $39(43.3)$ & NS & $13(46.4)$ & NS & NS \\
\hline \multicolumn{12}{|l|}{ Age, $N=347$} \\
\hline Median (IQR) & $33(27-40)$ & NS & $34(27-40)$ & NS & $34(27-44)$ & NS & $32(28-38)$ & NS & $33(29-40)$ & NS & NS \\
\hline \multicolumn{12}{|l|}{$\mathrm{CD} 4 / \mu \mathrm{l}, N=362$} \\
\hline Median (IQR) & $351(240-567)$ & NS & $310(146-310)$ & NS & $335(120-510)$ & NS & $335(222-535)$ & NS & $293(141-571)$ & NS & NS \\
\hline \multicolumn{12}{|l|}{ Viral load, $N=363$} \\
\hline Median log c/ml (IQR) & $4.54(3.85-5.25)$ & NS & $4.35(3.72-4.86)$ & NS & $4.82(4.22-5.28)$ & 0.045 & $4.64(3.88-5.10)$ & NS & $4.51(3.82-5.00)$ & NS & NS \\
\hline \multicolumn{12}{|l|}{ Tropisme G2P, N= 366} \\
\hline X4 (G2P $10 \%), N(\%)$ & $16(21.6)$ & NS & $7(11.1)$ & 0.014 & $30(45.5)$ & $<0.001$ & $17(17.0)$ & 0.097 & $14(22.2)$ & NS & $<0.001$ \\
\hline X4 (G2P 5,75 \%), N(\%) & $8(10.8)$ & NS & $3(4.8)$ & 0.014 & $24(36.4)$ & $<0.001$ & $9(9.0)$ & 0.057 & $10(15.9)$ & NS & $<0.001$ \\
\hline \multicolumn{12}{|l|}{ Tropisme PSSM, N= 360} \\
\hline X4 (PSSM-B), N(\%) & $12(16.9)$ & NS & $8(12.7)$ & 0.072 & $26(40.0)$ & $<0.001$ & $16(16.3)$ & NS & $14(22.2)$ & NS & 0.001 \\
\hline X4 (PSSM-C), N(\%) & & & $16(16,3)$ & NS & & & & & & & \\
\hline Tropisme Phenoseq, N= 277 & $8(12.9)$ & 0.021 & $19(31.1)$ & NS & $13(21.2)$ & NS & $26(28.0)$ & NS & & & NS \\
\hline \multicolumn{12}{|l|}{$X 4, N(\%)$} \\
\hline Transmission route & & & & & & & & & & & 0.005 \\
\hline Hetero, N (\%) & $44(59.5)$ & NS & $37(58.7)$ & NS & $42(63.6)$ & NS & $57(57.0)$ & NS & 39 (61.9) & NS & \\
\hline MSM, N (\%) & $0(0)$ & 0.003 & $3(4.8)$ & NS & $12(18.2)$ & 0.011 & $11(11.0)$ & NS & $6(9.5)$ & NS & \\
\hline Other, N (\%) & $9(12.2)$ & 0.020 & $2(3.2)$ & NS & $2(3.0)$ & NS & $4(4.0)$ & NS & $5(7.9)$ & NS & \\
\hline Unknown, N (\%) & $21(28,4)$ & NS & $21(33,3)$ & NS & $10(15,2)$ & 0,034 & $28(28,0)$ & NS & $13(20,6)$ & NS & \\
\hline Country of origin, $N=366$ & & & & & & & & & & & $<0.001$ \\
\hline Europe-West, N (\%) & $12(16.2)$ & 0.008 & $15(23.8)$ & NS & $31(47.0)$ & 0.001 & $22(22.0)$ & 0.053 & $27(42.9)$ & 0.016 & \\
\hline Europe-East, N (\%) & $13(17.6)$ & $<0.001$ & $0(0)$ & NS & $0(0)$ & 0.082 & $0(0)$ & 0.014 & $1(2.4)$ & NS & \\
\hline $\begin{array}{l}\text { Africa-Nord/Middle } \\
\text { East, N (\%) }\end{array}$ & $2(2.7)$ & NS & $1(1.6)$ & NS & $0(0)$ & NS & $4(4.0)$ & NS & $1(1.6)$ & NS & \\
\hline Africa-West, N (\%) & $10(13.5)$ & 0.016 & $1(1.6)$ & $<0.004$ & $2(3.0)$ & $<0.001$ & $58(58.0)$ & $<0.001$ & $20(31.7)$ & NS & \\
\hline Africa-Central, N (\%) & $26(35.1)$ & $<0.001$ & $19(30.2)$ & 0.001 & $1(1.5)$ & $<0.001$ & $7(7.0)$ & 0.003 & $7(11.1)$ & NS & \\
\hline Africa-East, N (\%) & $2(2.7)$ & NS & $8(12.7)$ & 0.002 & $0(0)$ & 0.050 & $2(2.2)$ & NS & $4(6.3)$ & NS & \\
\hline Africa-South, N (\%) & $2(2.7)$ & NS & $11(17.5)$ & $<0.001$ & $0(0)$ & NS & $0(0)$ & 0.023 & $0(0)$ & NS & \\
\hline
\end{tabular}


Table 2 Patient demographics, viral load, CD4 count, co-receptor tropism and transmission route for the most prevalent subtypes and CRF (Continued)

\begin{tabular}{|c|c|c|c|c|c|c|c|c|c|c|}
\hline Asia, N (\%) & $0(0)$ & 0.004 & $2(3.2)$ & NS & $29(43.9)$ & $<0.001$ & $0(0)$ & $<0.001$ & $0(0)$ & 0.007 \\
\hline Latin America, N (\%) & $0(0)$ & NS & $0(0)$ & NS & $0(0)$ & NS & $1(1)$ & NS & $1(1.6)$ & NS \\
\hline Unknown, N (\%) & $7(9.5)$ & NS & $6(9.5)$ & NS & $3(4.5)$ & NS & $6(6.0)$ & NS & $2(3.2)$ & NS \\
\hline
\end{tabular}

Subtype classification based on $p o l$ sequences

Significance was set at $\leq 0,05 ; p$-values $\leq 0,1$ are shown; $p$-values of $\leq 0,05$ are marked in bold; NS = not significant, $p>0,1$ 
from Central, East and South Africa with subtype C. Native Western Europeans accounted for between $16.2 \%$ and $47.0 \%$ of all non-B infections. They were underrepresented in subtype A $(p=0.008)$ and overrepresented in CRF01_AE $(p=0.001)$ and in the pooled group of other subtypes and CRFs $(p=0.016)$. MSM as route of transmission was the most frequently recorded for CRF01_AE $(18.2 \% ; p=0.011)$ but was never reported for subtype A (0 \%; $p=0.003)$. IVDU transmission was only reported by patients originating from Eastern Europe and all but one were subtype A infections. CXCR4-using viruses were significantly more prevalent in CRF01_AE $(p<0.001)$ and less prevalent in subtype $C(p=0.014)$ when using G2P or PSSM but not when using Phenoseq. The Phenoseq prediction system on the other hand reported a significantly lower CXCR4-use prevalence in subtype A $(p=0.022)$.

The analysis presented in Table 2 was repeated after grouping the patients based on env subtyping. The outcome was comparable (results not shown).

\section{Characteristics of the non- $B$ infections in the native population}

The majority (91.6 \%) of the Western-Europeans in the cohort were of Belgian or Luxembourg origin. The subtype distribution amongst this native population was CRF01_AE (30.6 \%), CRF02_AG (19.4\%), C (15.3\%), A (12.2\%), F (8.2 \%), CRF06 (2.0\%), D (1\%), G (1\%), H (1\%), and unassigned $(9.2 \%)$. The main infection route was heterosexual (68.1\%) followed by MSM (28.6 \%) and blood transfusion $(3.3 \%)$.

\section{Discussion}

Several Western European countries, especially those with large sub-Saharan African immigrant communities, have a high burden of HIV-1 non-B infections. The vast majority of these infections are acquired through heterosexual contacts. This contrasts with the subtype B epidemic in the same countries, that is driven predominantly by MSM and affects primarily young white men $[35,36]$. Increasing frequencies of non-B infections over time have been reported for Belgium [4], Spain [37], Italy [38], France [39], Sweden [40, 41] and Luxembourg [21]. Although information on the extent to which the subtype $B$ and non- $B$ epidemics intermingle remains limited, ascending prevalence's of non-B subtypes in native Europeans have been reported [21, 37, 41].

For this study we selected 3 sites in Belgium and Luxembourg, two small countries with a high migration rate, for investigation of the non- $B$ infections. Although the study is biased by the random selection of non-B infections and the unequal distribution of patients across the territory and can therefore not be seen as representative for the general epidemic in these countries, it does provide some interesting insights in the dynamics of the non-B infections. Important strengths of the study are the large sample size, the high subtype and CRF heterogeneity and the availability of both $p o l$ and $e n v$ sequences for subtype assignment.

A higher than expected percentage of patients with non-B infections were of Western European origin (30.7 \%; $24.5 \%$ from Belgium or Luxembourg and $5.6 \%$ from other Western European countries). While heterosexual transmission accounted for the majority (79.3\%) of infections a significant proportion of the patients reported MSM transmission (13.3\%). MSM infections were more frequent in Western Europeans $(28.6 \%$ versus $5.1 \%$ non-Western Europeans; $p<0.001$ ) providing proof for the spread of non- $\mathrm{B}$ subtypes to the native high-at-risk MSM population. One MSM transmission cluster of CRF02_AG was identified but this was the only clear phylogenetic indication of local non- $\mathrm{B}$ transmission in MSM. CRF01_AE was the most represented non-B subtype in Western Europeans (38.6\%), followed by CRF02_AG (27.7 \%), C (19.3\%) and A (14.5\%). The higher prevalence of CRF01_AE in individuals born in Western Europe can have several reasons. The Philippines and Thailand, where CRF01_AE is the major subtype [42] are in the top 5 of bride-donorcountries of Belgium (http://www.esf-agentschap.be/sites/ default/files/attachments/articles/eindrapport_partnermigratie.pdf). Sex tourism to South-East Asia may also have triggered the presence of CRF01_AE, in heterosexuals as well as MSM. MSM were significantly more represented amongst the CRF01_AE infections compared to the other subtypes. The profound intermingling of the CRF01_AE sequences from native Western Europeans and Asians and the lack of clear indications for onward local CRF01_AE transmission apart from some paired transmissions suggest that most CRF01_AE infections are imported infections.

Infection through IVDU was reported infrequently and only in Eastern-European individuals. The phylogenetic analysis revealed a very close genetic relationship between the viruses isolated from these Eastern-European IVDU but no indications for a spread of these particular strains to the local population.

Weakening of the association between subtypes and patient origin as well as between subtypes and exposure group has been reported before for the UK, Spain, Italy and Sweden [41, 43-46]. Future surveillance of this evolution will be important because this tendency can have implications on many facets of HIV care and prevention. Introduction of non-B subtypes in native high-at-risk populations may increase the overall genetic heterogeneity and the frequency of intersubtype recombination. Recombination may occasionally result in viral variants with increased pathogenesis as demonstrated recently in Cuba [47]. In our study, indications for novel intersubtype 
recombination were found in $6.3 \%$ of the patients and a high contribution of non- $\mathrm{B} / \mathrm{B}$ recombinants was noticed (5 of the 20 recombinants). This number of novel non- $\mathrm{B} / \mathrm{B}$ recombinants is almost certainly an underestimation as the initial selection of non- $B$ infections was based on the pol subtype and excluded infections classified as subtype B in pol. High percentages of novel recombinants have been reported for Spain (13.4\%) [48], Italy (9.4) [38] and UK (9.9\%) [49]. In these studies the classification of novel recombination is based only on the pol sequence. In our study however, 25 of the $49 \mathrm{pol}$ sequences considered as having a complex mosaic genetic pattern, were assigned A-like in env and classified as subtype A by pol phylogenetic analysis. Adequate classification of subtype A and CRF02_AG pol sequences seems particularly challenging because of the overall high genetic variability within these subtypes. This apparently may lead to a false identification of novel recombinants and may partly explain the important differences in frequency of novel recombination between studies, a hypothesis that needs to be confirmed. Local spread of these novel recombinants was still limited, only one small transmission cluster of a novel A/B recombinant was observed.

The patients infected with the most represented subtypes, A, C, CRF01_AE and CRF02_AG, did not differ in age, gender or $\mathrm{CD} 4$ count. The significant differences in distribution of patient origin followed the geographic distribution pattern of the respective subtypes [2].

The higher prevalence of CXCR4-use in CRF01_AE when using the G2P or PSSM prediction algorithms and the lower prevalence of CXCR4-use in subtype C confirmed previous findings [10,11, 14, 50, 51]. It is known however that G2P and PSSM lack accuracy for non-B subtypes [14, 52, 53]. Recently, an algorithm has been developed that claims high sensitivity and specificity for the subtypes A, B, C, D, CRF01_AE and CRF02_AG [30]. When applying this algorithm the prevalences of CXCR4use for the subtypes A and CRF01_AE differed considerably from the ones recorded with G2P and PSSM. These observations show that problems with co-receptor tropism prediction of some non-B subtypes are still unresolved and that urgent actions for improvements are needed.

We were unable to define subtype related differences in viral load or CD4 count but have to acknowledge that it is a shortcoming of this study that viral load and CD4 data were based on single measurement and could not be corrected for time of infection.

\section{Conclusion}

A higher than expected proportion of the non-B infections sampled in Belgium and Luxembourg were diagnosed in individuals of Western European origin. The spread of African and Asian subtypes to the local population resulted from heterosexual as well as MSM transmission.
Local spread remains limited. Subtype related differences in patient origin, infection route and CXCR4-use were noticed but no differences in gender, age, CD4 count or viral load were found. Future monitoring of the introduction of non-B infections in the local population is warranted both from an epidemiological and a prevention perspective.

\section{Availability of supporting data}

Sequences were submitted to the National Center for Biotechnology Information (NCBI) Genbank and are available under accession numbers KT863537 to KT863 946 (protease sequences), KT863947 to KT864356 (reverse transcriptase sequences) and KT864357 to KT864 674 (envelope sequences).

\section{Abbreviations}

HIV: Human Immunodeficiency Virus; CCR5: Chemokine (C-C motif) Receptor type 5; CXCR4: Chemokine (C-X-C motif) Receptor type 4; Pol: Polymerase; Env: Envelope; MSM: Men having sex with men; CRF: Circulating recombinant from; Nt: Nucleotide; IDNS: Integrated database network system; V3: Variable region 3; PSSM: Position specific scoring matrix; G2P: Geno-to-Pheno; SCUEAL: Subtype classification using evolutionary algorithms; jpHMM: Jumping profile hidden markov model; NCBI: National center for biotechnology; aLRT: Approximate Likelihood Ratio Test; PhyML: Maximum likelihood phylogenies; SPSS: Statistical package for the social sciences; IBM: International business machines corporation; IVDU: Intravenous drug use.

\section{Competing interests}

The authors declare that they have no competing interests.

\section{Authors' contribution}

KD and CV coordinated the data collection and analyses and wrote the manuscript. DPB, CSD and KF contributed to the analyses and preparation of the manuscript, KD, VM and MS performed the practical work, AVDH and JYS contributed samples and clinical data, VM performed the statistical analyses. All authors have read and approved the final manuscript.

\section{Financial support}

The Aids Reference Laboratories of Ghent and Antwerp are supported by the Belgian Ministry of Social Affairs through a fund within the Health Insurance System.

The Laboratory of Retrovirology of Luxembourg is supported by the Fondation Recherche sur le SIDA of Luxembourg.

\section{Author details}

${ }^{1}$ Aids Reference Laboratory, Department of Clinical Chemistry, Microbiology and Immunology, Ghent University, De Pintelaan 185-Blok A, B-9000, Ghent, Belgium. ${ }^{2}$ Aids Reference laboratory, Department of Clinical Sciences,

Institute of Tropical Medicine, Nationalestraat 155, B-2000 Antwerp, Belgium. ${ }^{3}$ Laboratory of Retrovirology, Department of Infection and Immunity, Luxembourg Institute of Health, Val Fleuri 84, L-1526 Luxembourg, Luxembourg.

Received: 7 May 2015 Accepted: 14 October 2015

Published online: 16 November 2015

\section{References}

1. Robertson DL, Anderson JP, Bradac JA, Carr JK, Foley B, Funkhouser RK, et al. HIV-1 nomenclature proposal. Science. 2000;288(5463):55-6.

2. Hemelaar J, Gouws E, Ghys PD, Osmanov S. C W-UNHI: global trends in molecular epidemiology of HIV-1 during 2000-2007. Aids. 2011;25(5):679-89.

3. Deroo S, Robert I, Fontaine E, Lambert C, Plesseria JM, Arendt V, et al. HIV-1 subtypes in Luxembourg, 1983-2000. Aids. 2002;16(18):2461-7.

4. Snoeck J, Van Laethem K, Hermans P, Van Wijngaerden E, Derdelinckx I, Schrooten $Y$, et al. Rising prevalence of HIV-1 non-B subtypes in Belgium: 1983-2001. J Acquir Immune Defic Syndr. 2004;35(3):279-85. 
5. Struck D, Roman F, De Landtsheer S, Servais JY, Lambert C, Masquelier C, et al. Near Full-Length Characterization and Population Dynamics of the Human Immunodeficiency Virus Type I Circulating Recombinant Form 42 (CRF42_BF) in Luxembourg. AIDS Res Hum Retroviruses. 2015;31(5):554-8.

6. Langford SE, Ananworanich J, Cooper DA. Predictors of disease progression in HIV infection: a review. AIDS Res Ther. 2007;4:11.

7. Bhargava M, Cajas JM, Wainberg MA, Klein MB, Pai NP. Do HIV-1 non-B subtypes differentially impact resistance mutations and clinical disease progression in treated populations? Evidence from a systematic review. J Int AIDS Soc. 2014;17:18944

8. Pasquier C, Millot N, Njouom R, Sandres K, Cazabat M, Puel J, et al. HIV-1 subtyping using phylogenetic analysis of pol gene sequences. J Virol Methods. 2001;94(1-2):45-54.

9. Neogi U, Bontell I, Shet A, De Costa A, Gupta S, Diwan V, et al. Molecular epidemiology of HIV-1 subtypes in India: origin and evolutionary history of the predominant subtype C. Plos One. 2012;7:6.

10. Chalmet K, Dauwe K, Foquet L, Baatz F, Seguin-Devaux C, Van der Gucht B, et al. Presence of CXCR4-using HIV-1 in patients with recently diagnosed infection: correlates and evidence for transmission. J Infect Dis. 2012;205(2): 174-84.

11. Phuphuakrat A, Phawattanakul S, Pasomsub E, Kiertiburanakul S, Chantratita W, Sungkanuparph S. Coreceptor tropism determined by genotypic assay in HIV-1 circulating in Thailand, where CRF01_AE predominates. Hiv Medicine. 2014;15(5):269-75.

12. Lin NH, Smeaton LM, Giguel F, Novitsky V, Moyo S, Mitchell RM, et al. Prevalence and clinical associations of CXCR4-using HIV-1 among treatment-naive subtype C-infected women in Botswana. J Acquir Immune Defic Syndr. 2011;57(1):46-50.

13. Huang W, Eshleman SH, Toma J, Fransen S, Stawiski E, Paxinos EE, et al. Coreceptor tropism in human immunodeficiency virus type 1 subtype $D$ : high prevalence of CXCR4 tropism and heterogeneous composition of viral populations. J Virol. 2007;81(15):7885-93.

14. Raymond S, Delobel P, Rogez S, Encinas S, Bruel P, Pasquier C, et al. Genotypic prediction of HIV-1 CRF01-AE tropism. J Clin Microbiol. 2013; 51(2):564-70.

15. Cecilia D, Kulkarni SS, Tripathy SP, Gangakhedkar RR, Paranjape RS, Gadkari DA. Absence of coreceptor switch with disease progression in human immunodeficiency virus infections in India. Virology. 2000;271(2): 253-8.

16. Bártolo I, Abecasis AB, Borrego P, Barroso H, McCutchan F, Gomes $P$, et al, Origin and epidemiological history of HIV-1 CRF14_BG. Plos One. 2011;6:9.

17. Li Y, Han Y, Xie J, Gu L, Li W, Wang H, et al. CRF01_AE subtype is associated with X4 tropism and fast HIV progression in Chinese patients infected through sexual transmission. AIDS. 2014;28(4):521-30.

18. Li X, Xue Y, Zhou L, Lin Y, Yu X, Wang X, et al. Evidence that HIV-1 CRF01_AE is associated with low CD4 + T cell count and CXCR4 co-receptor usage in recently infected young men who have sex with men (MSM) in Shanghai, China. Plos One. 2014;9:2.

19. Koot M, Keet IP, Vos AH, de Goede RE, Roos MT, Coutinho RA, et al. Prognostic value of HIV-1 syncytium-inducing phenotype for rate of CD4+ cell depletion and progression to AIDS. Ann Intern Med. 1993;118(9):681-8.

20. Connor RI, Sheridan KE, Ceradini D, Choe S, Landau NR. Change in coreceptor use correlates with disease progression in HIV-1-infected individuals. J Exp Med. 1997;185(4):621-8.

21. Struck D, Lawyer G, Ternes A-MM, Schmit J-CC, Bercoff DP. COMET: adaptive context-based modeling for ultrafast HIV-1 subtype identification. Nucleic Acids Res. 2015;42:18.

22. Verhofstede C, Brudney D, Reynaerts J, Vaira D, Fransen K, De Bel A, et al. Concordance between HIV-1 genotypic coreceptor tropism predictions based on plasma RNA and proviral DNA. Hiv Med. 2011;12(9):544-52.

23. de Oliveira T, Deforche K, Cassol S, Salminen M, Paraskevis D, Seebregts C, et al. An automated genotyping system for analysis of HIV-1 and other microbial sequences. Bioinformatics. 2005;21(19):3797-800.

24. Pond SLK, Posada D, Stawiski E, Chappey C, Poon AFY, Hughes G, et al. An evolutionary model-based algorithm for accurate phylogenetic breakpoint mapping and subtype prediction in HIV-1. PLoS Comput Biol. 2009:5:11.

25. Schultz AK, Zhang M, Bulla I, Leitner T, Korber B, Morgenstern B, et al. jpHMM: improving the reliability of recombination prediction in HIV-1 (vol 37, pg W647, 2009). Nucleic Acids Res. 2010;38(3):1059-9.
26. Rozanov M, Plikat U, Chappey C, Kochergin A, Tatusova T. A web-based genotyping resource for viral sequences. Nucleic Acids Res. 2004;32:W654-9.

27. Sing T, Low AJ, Beerenwinkel N, Sander O, Cheung PK, Domingues FS, et al. Predicting HIV coreceptor usage on the basis of genetic and clinical covariates. Antivir Ther. 2007;12(7):1097-106.

28. Jensen MA, Li FS, van 't Wout AB, Nickle DC, Shriner D, He HX, et al. Improved coreceptor usage prediction and genotypic monitoring of R5-toX4 transition by motif analysis of human immunodeficiency virus type 1 env V3 loop sequences. J Virol. 2003;77(24):13376-88.

29. Jensen MA, Coetzer M, van 't Wout AB, Morris $L$, Mullins JI. A reliable phenotype predictor for human immunodeficiency virus type 1 subtype $C$ based on envelope V3 sequences. J Virol. 2006;80(10):4698-704.

30. Cashin K, Gray LR, Harvey KL, Perez-Bercoff D, Lee GQ, Sterjovski J, et al. Reliable genotypic tropism tests for the major HIV-1 subtypes. Scientific Reports. 2015;5:8543.

31. Darriba D, Taboada GL, Doallo R, Posada D. jModelTest 2: more models, new heuristics and parallel computing. Nat Methods. 2012;9(8):772.

32. Guindon S, Delsuc F, Dufayard JF, Gascuel O. Estimating maximum likelihood phylogenies with PhyML. Methods Mol Biol. 2009;537:113-37.

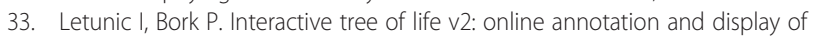
phylogenetic trees made easy. Nucleic Acids Res. 2011;39(Web Server issue): W475-478.

34. Ragonnet-Cronin M, Hodcroft E, Hue S, Fearnhill E, Delpech V, Brown AJ, et al. Automated analysis of phylogenetic clusters. Bmc Bioinformatics. 2013; 14:317.

35. Abecasis AB, Wensing AM, Paraskevis $D$, Vercauteren J, Theys $K$, Van de Vijver DA, et al. HIV-1 subtype distribution and its demographic determinants in newly diagnosed patients in Europe suggest highly compartmentalized epidemics. Retrovirology. 2013;10:7.

36. Chalmet K, Staelens D, Blot S, Dinakis S, Pelgrom J, Plum J, et al. Epidemiological study of phylogenetic transmission clusters in a local HIV-1 epidemic reveals distinct differences between subtype $B$ and non-B infections. BMC Infect Dis. 2010;10:262.

37. Holguin A, de Mulder M, Yebra G, Lopez M, Soriano V. Increase of non-B subtypes and recombinants among newly diagnosed HIV-1 native Spaniards and immigrants in Spain. Curr HIV Res. 2008;6(4):327-34.

38. Lai A, Riva C, Marconi A, Balestrieri M, Razzolini F, Meini G, et al. Changing patterns in HIV-1 non-B clade prevalence and diversity in Italy over three decades. HIV Med. 2010;11(9):593-602.

39. Descamps D, Chaix ML, Montes B, Pakianather S, Charpentier C, Storto A, et al. Increasing prevalence of transmitted drug resistance mutations and non-B subtype circulation in antiretroviral-naive chronically HIV-infected patients from 2001 to 2006/2007 in France. J Antimicrob Chemother. 2010; 65(12):2620-7.

40. Sonnerborg A, Durdevic S, Giesecke J, Sallberg M. Dynamics of the HIV-1 subtype distribution in the Swedish HIV-1 epidemic during the period 1980 to 1993. AIDS Res Hum Retroviruses. 1997;13(4):343-5.

41. Neogi U, Haggblom A, Santacatterina M, Bratt G, Gisslen M, Albert J, et al. Temporal trends in the swedish HIV-1 epidemic: increase in Non-B subtypes and recombinant forms over three decades. Plos One. 2014;9:6.

42. Angelis $\mathrm{K}$, Albert J, Mamais I, Magiorkinis G, Hatzakis A, Hamouda O, et al. Global Dispersal Pattern of HIV Type 1 Subtype CRF01_AE: A Genetic Trace of Human Mobility Related to Heterosexual Sexual Activities Centralized in Southeast Asia. J Infect Dis. 2015;211(11):1735-44.

43. Aggarwal I, Smith M, Tatt ID, Murad S, Osner N, Geretti AM, et al. Evidence for onward transmission of HIV-1 non-B subtype strains in the United Kingdom. J Acquir Immune Defic Syndr. 2006;41(2):201-9.

44. Gonzalez-Alba JM, Holguin A, Garcia R, Garcia-Bujalance S, Alonso R, Suarez A, et al. Molecular surveillance of HIV-1 in Madrid, Spain: a phylogeographic analysis. J Virol. 2011;85(20):10755-63.

45. Bracho MA, Sentandreu V, Alastrue I, Belda J, Juan A, Fernandez-Garcia E, et al. Emerging trends in CRF02_AG variants transmission among Men Who have Sex with Men in Spain. J Acquir Immune Defic Syndr. 2014;65(3):E130-3.

46. Giuliani M, Montieri S, Palamara G, Latini A, Alteri C, Perno CF, et al. Non-B HIV type 1 subtypes among Men Who have Sex with Men in Rome, Italy. AIDS Res Hum Retroviruses. 2009;25(2):157-64.

47. Kouri V, Khouri R, Alemán Y, Abrahantes Y, Vercauteren J, Pineda-Peña A-C, et al. CRF19_cpx is an Evolutionary fit HIV-1 Variant Strongly Associated With Rapid Progression to AIDS in Cuba. EBioMedicine 2015;2:244-54.

48. Yebra G, de Mulder M, Perez-Elias MJ, Perez-Molina JA, Galan JC, Llenas-Garcia J, et al. Increase of transmitted drug resistance among HIV- 
infected Sub-Saharan Africans residing in Spain in contrast to the native population. Plos One. 2011;6:10.

49. Dunn D, Drug UCGH. The increasing genetic diversity of HIV-1 in the UK, 2002-2010. Aids. 2014;28(5):773-80.

50. Jakobsen MR, Cashin K, Roche M, Sterjovski J, Ellett A, Borm K, et al. Longitudinal analysis of CCR5 and CXCR4 usage in a cohort of antiretroviral therapy-naive subjects with progressive HIV-1 subtype $\mathrm{C}$ infection. Plos One. 2013;8(6):e65950.

51. Ping LH, Nelson JAE, Hoffman IF, Schock J, Lamers SL, Goodman M, et al. Characterization of V3 sequence heterogeneity in subtype $\mathrm{C}$ human immunodeficiency virus type 1 isolates from Malawi: underrepresentation of X4 variants. J Virol. 1999;73(8):6271-81.

52. Mulinge M, Lemaire M, Servais JY, Rybicki A, Struck D, da Silva ES, et al. HIV1 tropism determination using a phenotypic Env recombinant viral assay highlights overestimation of CXCR4-usage by genotypic prediction algorithms for CRRF01_AE and CRF02_AG. Plos One. 2013;8:5.

53. Bartlett AD, MaCartney MJ, Conibear TC, Feyertag F, Smith CJ, Johnson MA et al. The utility of different bioinformatics algorithms for genotypic HIV-1 tropism testing in a large clinical cohort with multiple subtypes. AIDS. 2014; 28(11):1611-7.

\section{Submit your next manuscript to BioMed Central and take full advantage of:}

- Convenient online submission

- Thorough peer review

- No space constraints or color figure charges

- Immediate publication on acceptance

- Inclusion in PubMed, CAS, Scopus and Google Scholar

- Research which is freely available for redistribution 